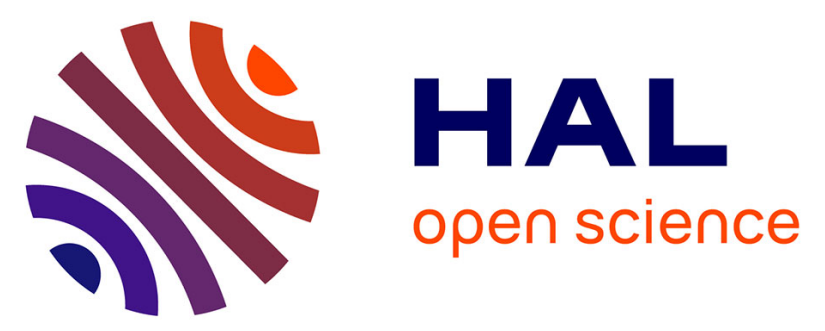

\title{
How Dark Are Radial Breathing Modes in Plasmonic Nanodisks?
}

Franz-Philipp Schmidt, Arthur Losquin, Ferdinand Hofer, Andreas Hohenau, Joachim R Krenn, Mathieu K Kociak

\section{- To cite this version:}

Franz-Philipp Schmidt, Arthur Losquin, Ferdinand Hofer, Andreas Hohenau, Joachim R Krenn, et al. How Dark Are Radial Breathing Modes in Plasmonic Nanodisks?. ACS photonics, 2018, 5 (3), pp.861-866. 10.1021/acsphotonics.7b01060 . hal-01804349

\section{HAL Id: hal-01804349 \\ https://hal.science/hal-01804349}

Submitted on 31 May 2018

HAL is a multi-disciplinary open access archive for the deposit and dissemination of scientific research documents, whether they are published or not. The documents may come from teaching and research institutions in France or abroad, or from public or private research centers.
L'archive ouverte pluridisciplinaire $\mathbf{H A L}$, est destinée au dépôt et à la diffusion de documents scientifiques de niveau recherche, publiés ou non, émanant des établissements d'enseignement et de recherche français ou étrangers, des laboratoires publics ou privés.

\section{(1)(1) $\$(0)$}

Distributed under a Creative Commons Attribution - NonCommercial - ShareAlikel 4.0 


\title{
How Dark Are Radial Breathing Modes in Plasmonic Nanodisks?
}

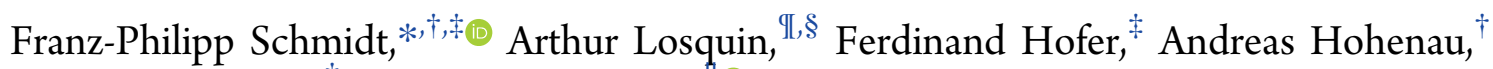 \\ Joachim R. Krenn, ${ }^{\dagger}$ and Mathieu Kociak ${ }^{1 \odot}$ \\ ${ }^{\dagger}$ Institute of Physics, University of Graz, Graz 8010, Austria \\ ${ }^{\ddagger}$ Institute for Electron Microscopy and Nanoanalysis, Graz University of Technology, Graz 8010, Austria \\ II Department of Physics, Lund University, Lund 22100 , Sweden \\ ${ }^{\S}$ Laboratoire Ondes et Matière d'Aquitaine, UMR 5798, CNRS University of Bordeaux, F 33405 Talence Cedex, France \\ "Laboratoire de Physique des Solides, CNRS UMR 8502, Université Paris Sud, 91405 Orsay, France
}

\begin{abstract}
Due to a vanishing dipole moment, radial breathing modes in small flat plasmonic nanoparticles do not couple to light and have to be probed with a near field source, as in electron energy loss spectroscopy (EELS). With increasing particle size, retardation gives rise to light coupling, enabling probing breathing modes optically or by cathodoluminescence (CL). Here, we investigate single silver nanodisks with diameters of $150-500 \mathrm{~nm}$ by EELS and CL in an electron microscope and quantify the EELS/CL ratio, which corre

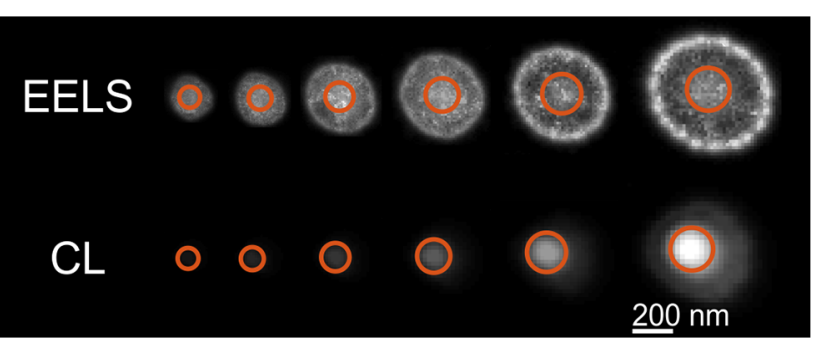
sponds to the ratio of full to radiative damping of the breathing mode. For the investigated diameter range, we find the CL signal to increase by about 1 order of magnitude, in agreement with numerical simulations. Due to reciprocity, our findings corroborate former optical experiments and enable a quantitative understanding of the light coupling of dark plasmonic modes.
\end{abstract}

KEYWORDS: plasmonics, electron energy loss spectroscopy, cathodoluminescence, transmission electron microscopy, nanoparticles

In recent years, focused electron probes have opened new avenues toward the spectral analysis of nanostructures with broadband optical sensitivity. Electron energy loss spectrosco py (EELS) in a scanning transmission electron microscope (STEM) (see Figure 1a) provides information with nanometer spatial resolution and sub eV spectral resolution. The EELS signal is proportional to the photonic local density of states (LDOS), projected along the electron beam direction. ${ }^{1,2}$ EELS thus provides access to all linear optical properties of nano objects and has been applied to probe highly localized fields, in particular plasmon eigenmodes in nanostructures. ${ }^{3,4}$ Thanks to the unprecedented spatial resolution of STEM EELS, the classification of plasmon modes on flat nanoparticles into edge and film modes could be derived ${ }^{5}$ and their correspondence to the dispersion relations of straight edges and extended films, respectively, was found. 6,7 The introduction of 3D EELS imaging based on tomographic reconstruction has further enriched the field. $^{8-10} \mathrm{~A}$ related technique is cathodolumi nescence $(\mathrm{CL}),{ }^{11}$ relying on photon emission upon electron excitation (Figure 1a). In a STEM, CL probes plasmon eigenmodes with the same high spatial resolution as EELS and even better energy resolution that is not limited by the exciting electron beam. ${ }^{12} \mathrm{CL}$ is related to the radiative part of the LDOS. $^{11,13}$ Therefore, the comparison of EELS and CL provides complementary information on the optical properties of plasmonic systems ${ }^{13-15}$ that can be related to extinction and scattering cross sections, respectively. ${ }^{16,17}$ This, in turn, is closely related to the respective contributions to damping in the system in terms of radiation damping and full damping, i.e., the sum of the radiation and ohmic contribution. ${ }^{18,19}$

In this Article, we discuss a combined EELS/CL study to characterize light coupling of radial breathing modes (RBMs) in silver nanodisks. RBMs have zero net dipole moment. In disks much smaller than the light wavelength, they are thus dark modes that do not couple to light. However, increasing size gives rise to retardation, thus making the RBM bright. ${ }^{20,21}$ We find that with increasing size $(150-500 \mathrm{~nm})$ the EELS signal does not change significantly, but the CL signal increases by about 1 order of magnitude, corresponding to increased scattering ${ }^{22}$ and thus radiation damping. To quantify the light coupling properties of RBMs, we introduce EELS/CL ratios that we obtain from different disk sizes and support the experimental results with numerical simulations. The direct measurement of the bright vs dark properties of plasmonic modes will benefit not only their fundamental understanding but also their use in processes such as surface enhanced fluorescence or surface enhanced Raman scattering and in applications such as biosensing. 


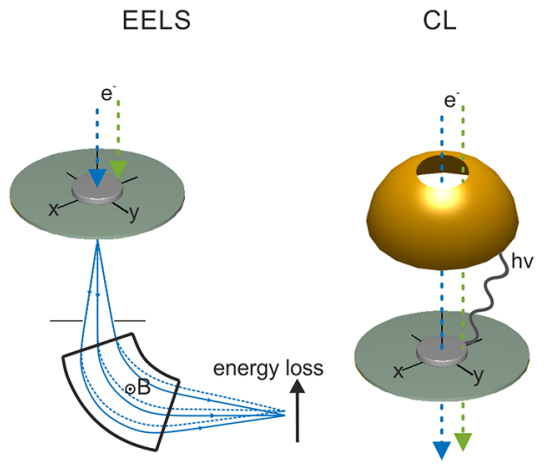

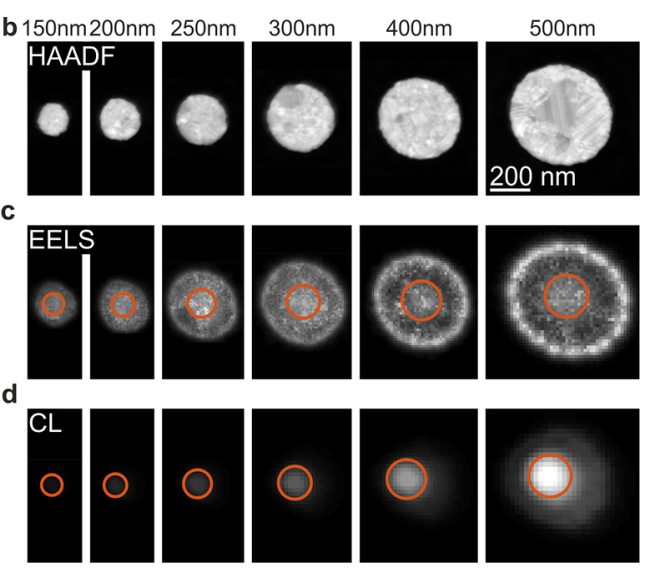

Figure 1. Experimental methods and spectroscopic maps of $30 \mathrm{~nm}$ thick silver disks with diameters of $150-500 \mathrm{~nm}$. (a) Sketches of experimental EELS and CL setups: In EELS, the energy lost by the electrons transmitted through the sample is analyzed by an electron spectrometer. In CL, the energy of light emitted from the sample is analyzed. The light is collected from above the sample within a limited detection range, which excludes the propagation direction of the electrons. (b) HAADF images, (c) EELS, and (d) CL maps of the RBM. The EELS and CL maps are integrated over an energy window of $\pm 0.25 \mathrm{eV}$ around the RBM peak energy, and the contrast range is normalized on the corresponding RBM peak areas (for details on the normalization see Figure S1a in the Supporting Information). The inner area of the orange circles indicates the region with the highest RBM signal.

\section{EXPERIMENTAL SECTION}

The nanodisks were fabricated by electron beam lithography on a $15 \mathrm{~nm}$ thick $\mathrm{Si}_{3} \mathrm{~N}_{4}$ substrate. The disk height was fixed at $30 \mathrm{~nm}$, and the diameter was varied between 150 and $500 \mathrm{~nm}$. We performed EELS and CL experiments on a VG STEM machine at $100 \mathrm{kV}$ acceleration voltage. All the data shown hereafter have been synthesized from EELS and CL spectral images taken on the same disks but at different times. For numerical simulations, we used the MNPBEM toolbox, ${ }^{23}$ which relies on the boundary element method. Details are given in the Methods section.

\section{RESULTS AND DISCUSSION}

Nanodisks possess high symmetry and are thus ideal geometries to investigate canonical plasmonic behavior. RBMs have a zero net dipole moment and are therefore dark modes (i.e., they do not couple to light) in disks much smaller than the light wavelength. Optical spectroscopy data have shown that for disks with a $200 \mathrm{~nm}$ diameter and inclined light incidence with respect to the disk surface, retardation allows RBM excitations by light, ${ }^{24}$ while no excitation was achieved for smaller disks. Considering the reciprocity principle, RBMs excited locally, e.g., by a fast electron beam, should therefore radiate light into the same inclined direction from where it could be excited optically, as long as a minimum nanodisk size (where retardation becomes relevant) is considered. We thus compare EELS and CL on single nanodisks with varying diameters to quantify the size dependent retardation effect and thus the light coupling properties of RBMs.

We start with a direct comparison of the RBM maps in Figure 1, for nanodisk diameters of 150-500 nm (high angle annular dark field, HAADF, images in Figure 1b). The EELS maps in Figure 1c show the RBM intensities (highest within the orange circle) that roughly remain the same for all disk sizes. We note that signals from neighboring plasmon modes contribute within the spectral window of the maps. These are higher order edge modes for the larger disks (i.e., periodically varying intensity along the circumference). For the smaller disks the surface plasmon $\mathrm{SP}_{1}$ (ref 6 and compare Figure 2) causes an additional signal homogeneously distributed over the
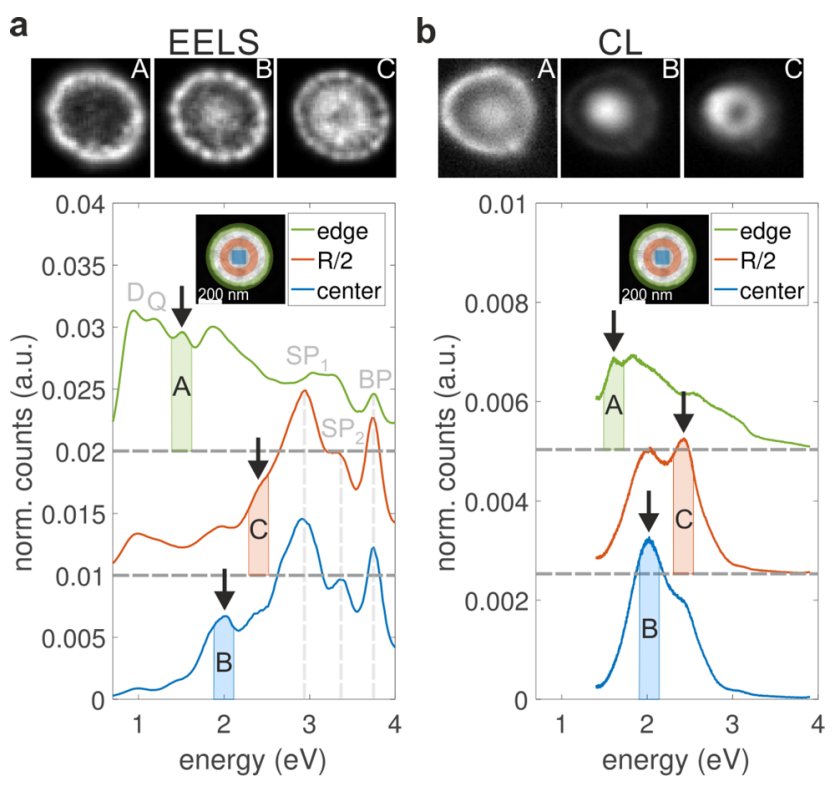

Figure 2. EELS vs CL measurements on a $500 \mathrm{~nm}$ diameter silver disk on a $\mathrm{Si}_{3} \mathrm{~N}_{4}$ membrane: (a) EELS and (b) CL maps and spectra. Maps $\mathrm{A}-\mathrm{C}$ (top) extracted at energies given by the black arrows in the spectra (bottom) and integrated over a constant energy width (colored area below the spectra) correspond to an edge (A), film (B), and mixed edge/film (C) mode with distinct mode pattern. The spectra originate from regions as indicated in the inset, i.e., edge (green), half radial distance (red), and center (blue) of the disk. D, Q $\mathrm{SP}_{1}, \mathrm{SP}_{2}$, and $\mathrm{BP}$ correspond to the dipolar, quadrupolar, antisymmetric surface, symmetric surface, and bulk plasmon mode, respectively.

disk surface, so that the RBM signal at the disk center is poorly visible. The orange circles delineate the RBM area to clearly separate it from other mode contributions. The behavior of the CL signal is markedly different: being virtually absent for the smallest disk, it strongly increases with increasing disk diameter (Figure 1d). The slight oval shape as well as the noncentric emission maximum for the 400 and $500 \mathrm{~nm}$ disks comes from a small sample drift. We discuss a more detailed interpretation 
a

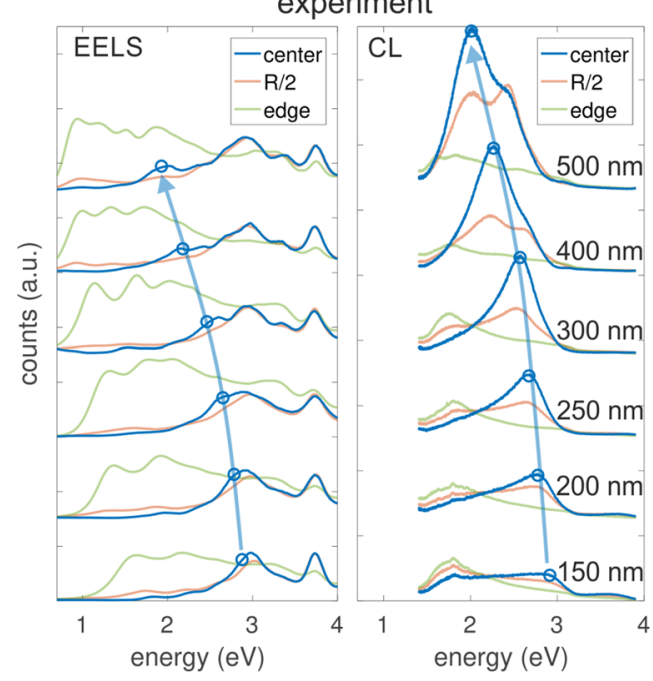

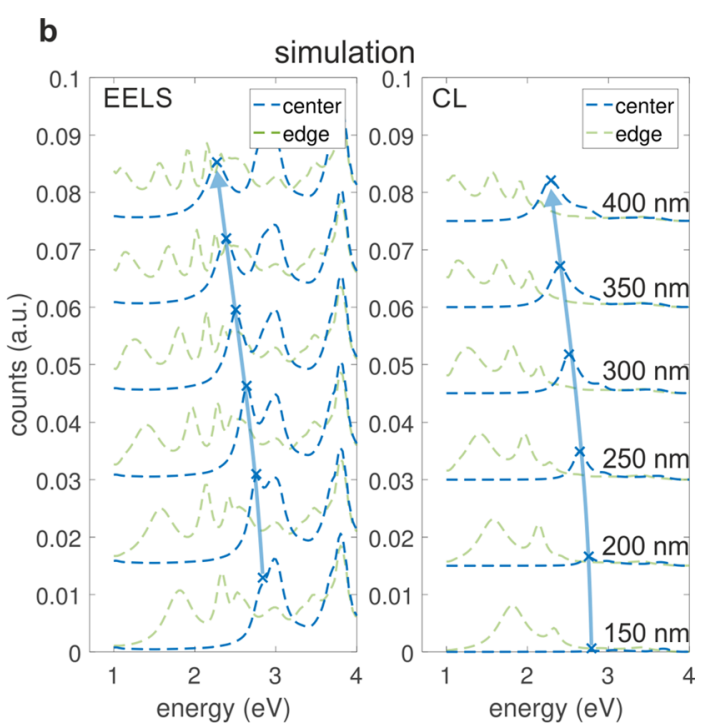

Figure 3. Size dependent EELS and CL spectra. (a) Experimental EELS and CL spectra of Ag disks with diameters as indicated. The spectra correspond to the center (blue), $R / 2$ (red), and edge (green) region of the disks. (b) Corresponding simulated EELS and CL spectra. The blue arrows are guides to the eye.

of the RBM maps in the next to last two paragraphs of this Article.

To understand this finding, we first include the full experimental spectral range in our analysis. For an example of a single silver nanodisk, $500 \mathrm{~nm}$ in diameter, the EELS (a) and CL (b) data are depicted in Figure 2. We observe that, first, EELS and CL maps at three different mode energies (AC) give similar mode patterns, as shown in the top row of Figure 2. While map A has the highest intensity along the disk circumference, mode $\mathrm{B}$ shows an additional feature in the center, and mode $\mathrm{C}$ exhibits a ring shaped intensity distribution at half radial distance $(R / 2)$. Second, EELS and $\mathrm{CL}$ spectra obtained from specific regions on the particle are quite similar. The green spectra correspond to the edge, the red spectra to the region at $R / 2$, and the blue spectra to the disk center, as indicated in the inset. Nonradiating modes are evidently missing in the CL data, which include the surface plasmons $\mathrm{SP}_{1}$ at $2.9 \mathrm{eV}$ (antisymmetric mode) and $\mathrm{SP}_{2}$ at 3.35 $\mathrm{eV}$ (symmetric mode) and the bulk plasmon $\mathrm{BP}$ at $3.75 \mathrm{eV}{ }^{6}$ Energies below $1.4 \mathrm{eV}$ could not be detected by the CL setup.

In accordance with former work ${ }^{5}$ we assign mode $A$ to an edge mode of third order (hexapole). The first and second order edge modes (dipole and quadrupole) are well resolved in the EELS data (green spectrum, D and Q) but are outside the $\mathrm{CL}$ detection range. Mode $\mathrm{B}$ is the RBM (a film mode of first order), and mode $\mathrm{C}$ is a mixed edge/film mode, also referred to as $(0,1)$ and $(1,1)$ modes, respectively. ${ }^{5}$ For both data sets, mode patterns and spectral signatures are thus mostly identical, in accordance with previous work on EELS and CL. ${ }^{16}$ Importantly, however, the RBM CL signal is quite strong (B, blue spectrum in Figure $2 \mathrm{~b}$ ), which can readily be assigned to retardation induced light coupling, given the disk diameter of $500 \mathrm{~nm}$.

Aiming at a quantization of the light coupling size dependence, we now trace the signal intensity of the RBM as a function of the disk diameters for EELS and CL. EELS and CL spectra for all nanodisk diameters $150-500 \mathrm{~nm}$ are shown in Figure 3. Experimental data, again from three different disk regions, i.e., disk center (blue), along the circumference at $R / 2$ (red), and the edge region (green), are displayed in Figure $3 a$.
For a detailed description of the spectrum normalization for the EELS and CL data see the Supporting Information ("Normalization" section). On one hand, the peak positions of the RBM shift in a similar manner for both EELS and CL, as indicated by the blue arrows. On the other hand, the different RBM peak intensities in EELS and CL are evident from the spectra. In particular, the CL RBM peak intensity strongly increases with increasing disk size, while EELS shows an almost constant RBM intensity for all disk diameters. We note that all plasmon eigenmodes discussed in this work are below 3 $\mathrm{eV}$, and therefore damping due to interband transitions is not to be expected. The simulations plotted in Figure $3 \mathrm{~b}$ reproduce the measured trend. While for a disk diameter of $400 \mathrm{~nm}$ an intense RBM peak is found in the simulated CL spectrum, the peak maximum is reduced by over $90 \%$ for the disk diameter of $150 \mathrm{~nm}$ (see Figure S1b in the Supporting Information). In addition, in the simulations the spectra originating from the disk edge (green spectra) clearly resolve the dipolar edge mode for EELS and CL (lowest energy peak in each spectrum), while the higher order edge modes are less pronounced in the case of CL. This is valid in particular for the smaller disk diameters, indicating the weakly radiating character for these modes. In accordance with the experimental setup, light detection is considered in the simulations in the half space above the sample, excluding the hole at the topmost position where the electron beam passes (see Methods). The agreement between experiment and theory but also the mismatch in particular for the CL edge spectra are further discussed in the Supporting Information (see "Agreement between simulation and experi ment" section and Figure S4). This includes spatial averaging, defects within the sample, and the lack of knowledge on the exact angular detection range as possible reasons for the mismatch in the CL edge spectra.

To further analyze the size dependent light coupling proper ties of RBMs, we plot the CL to EELS ratio of the RBM signal in Figure 4 for both simulated (dashed) and experimental (solid) data. The signals used for the ratio values are summarized in Figure S1. We apply for the experimental CL to EELS ratio values a modified EELS signal $I_{\text {center }}-I_{R / 2}$ (instead of $I_{\text {center }}$ only), which we obtain by subtracting the $R / 2$ 


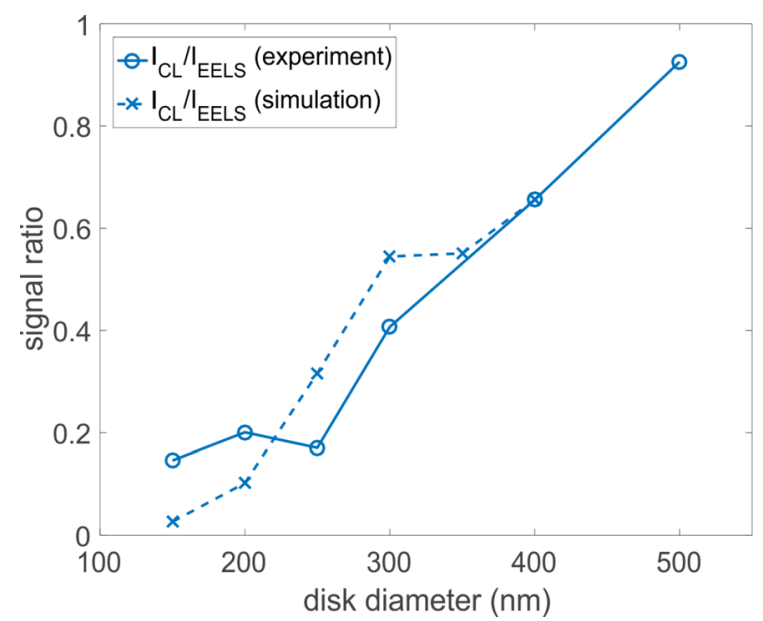

Figure 4. Ratio of CL to EELS signal of the RBM as a function of disk size for the experimental (solid) and simulated (dashed) data. Experimental ratios have been normalized so that the value for the disk diameter of $400 \mathrm{~nm}$ coincides with the simulated result.

spectrum from the center spectrum. This removes the contribution of the neighboring surface plasmon $\mathrm{SP}_{1}$ (compare red and blue EEL spectra in Figure 3a). Thereby, we extract an EELS signal for each disk diameter that originates from the RBM only. The experimental CL to EELS ratio values are renormalized to the simulated value of the $400 \mathrm{~nm}$ diameter disk, using the same normalization factor for all experimental ratio values. With the normalization, the experimental and the simulated data agree very well. We note that an alternative way to analyze the data is via the center to edge signal ratio, leading basically to the same results (Supporting Information, "Center to edge ratio", Figure S2). In any case, the CL to EELS ratio increases with disk diameter, showing a concurrent increase in scattering strength and radiation damping of the RBM. Increasing the nanodisk diameter from 150 to $500 \mathrm{~nm}$ this ratio increases by roughly 1 order of magnitude, in agreement with recent theoretical work on nanodisk RBMs. ${ }^{21}$ This clearly demonstrates that RBMs that are dark in small nanodisks can efficiently couple to light for larger disk sizes.

Finally, we discuss the direction of light emission from the nanodisk plasmon modes excited by fast electrons. Figure 5a shows two simulated CL spectra from the edge (green) and center (blue) regions of a silver nanodisk ( $30 \mathrm{~nm}$ thick, 200 $\mathrm{nm}$ in diameter, on a $15 \mathrm{~nm}$ thin $\mathrm{Si}_{3} \mathrm{~N}_{4}$ substrate modeled as another disk, $700 \mathrm{~nm}$ in diameter), where the light signal emitted in all directions ( $4 \pi \mathrm{sr}$ ) was considered (for simulations of other disk sizes see Figure S3 in the Supporting Information). To assign the plasmon modes to the peaks (1)(3), we plot in Figure $5 b$ the corresponding charge distribution. From that we attribute the dipolar, quadrupolar, and RBM mode to the peaks (1)-(3), respectively. Heuristically, EELS and CL probe the absolute value of the eigencharge distribution. ${ }^{14}$ For the RBM mode, the eigen charge density is much weaker on the edge than in the center, leading to an expected much stronger contrast in the center than on the edge, as confirmed by simulations in Figure 5b (3). Figure $5 \mathrm{c}$ illustrates the emission patterns of the modes by plotting the energy flux density of the electromagnetic field. We find that the dipole mode emission is fairly symmetric, while the quadrupolar mode shows two higher intensity lobes in the direction opposing the beam position, in accordance

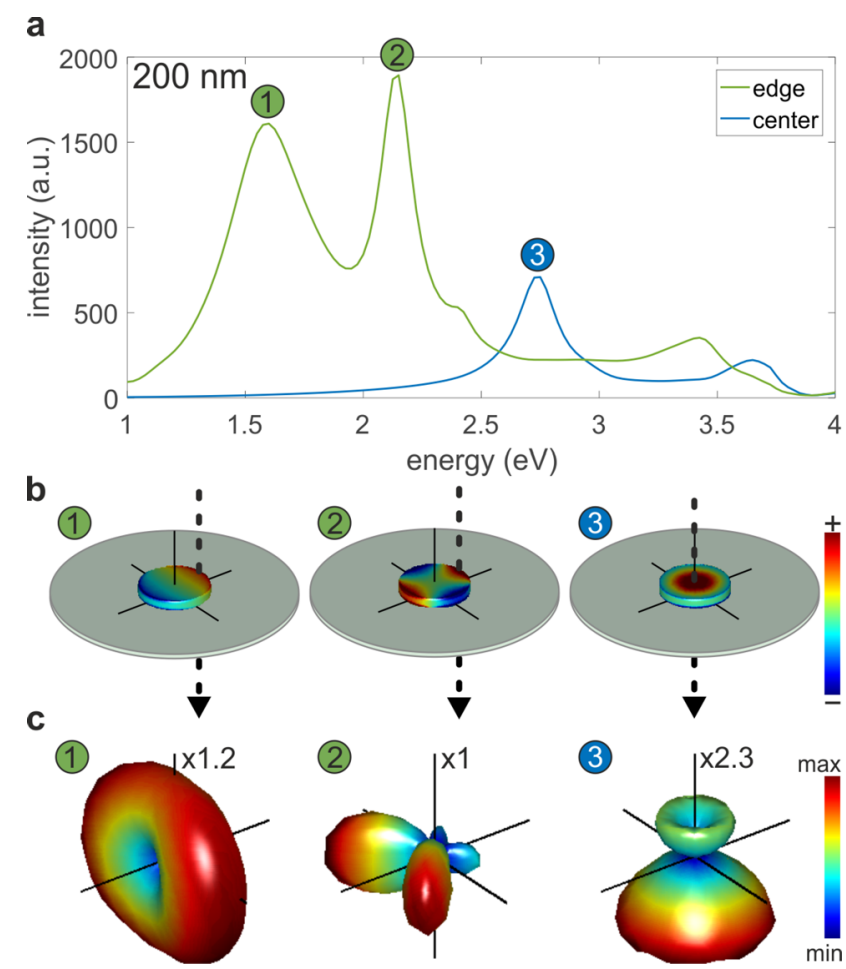

Figure 5. Light emission profiles from nanodisks. (a) Simulated CL spectra of a $30 \mathrm{~nm}$ thick Ag disk, $200 \mathrm{~nm}$ in diameter, from the edge (green) and the center (blue) region as indicated in (b). (b) Surface charge distribution of the dipolar (1), quadrupolar (2), and breathing mode (3), corresponding to the three peak energies in (a); the dashed arrows indicate position and direction of the exciting electron beam. (c) Emission patterns of the plasmon modes (1)-(3), with the color reflecting the absolute value of the energy flux density, each normalized to its maximum (red color). The relative intensity scale is given by the factors as depicted, indicating the lowest overall emission for the RBM (3).

with recently observed directional emission on gold nano disks. ${ }^{25}$ Similar to the RBM, the quadrupolar mode is of dark character for small particles, corresponding to smaller CL intensity for decreasing disk size (Figure S3). The RBM emits mostly into the substrate, with a maximum at a polar angle $\theta$ of $135^{\circ}$ and a weaker emission into the upper direction with an emission maximum at $\theta=45^{\circ}$. The RBM emission into the upper direction is strongly reduced for smaller disks, virtually disappearing for a disk diameter of $100 \mathrm{~nm}$ (see Figure S3 in the Supporting Information), in accordance with the small CL intensity measured for small disks. At $\theta=0^{\circ}$, i.e., in the direction perpendicular to the surface, there is no emission even for larger disk diameters, as expected from the RBM charge symmetry. It is thus evident that an RBM cannot be excited by a plane wave under perpendicular incidence for all disk sizes, while it can be excited by a plane wave with tilted incidence for large disks. In general, these results emphasize the reciprocity of data from CL and optical spectroscopy. ${ }^{24}$

It seems counterintuitive that the RBM, which for obvious symmetry reasons does not have a dipolar moment, can radiate. Indeed, as is the case for the edge modes, the most intense modes are usually dipolar in nature, and often, when the particle is small with respect to the light wavelength, only the dipolar radiation is detectable. This is because the multipolar decomposition in the far field corresponds to a polynomial series of terms with increasing power of products 
of $k$ and $R$, with $k=\omega / c$ and $R$ being the radius of the disk. For small $R$, only the dipolar component prevails so that in the case of the small disks, indeed, the RBM mode is very dark due to the missing dipolar contribution. As the size of the disk increases, the higher order components quickly increase, making the mode quite bright.

\section{CONCLUSIONS}

In summary, we have shown by a combined EELS/CL study how the dark mode character of plasmonic nanodisks is relieved with increasing disk diameter. Expressed as a CL/ EELS signal ratio, RBM light coupling increases for almost 1 order of magnitude when increasing the disk diameter from $150 \mathrm{~nm}$ to $500 \mathrm{~nm}$. Correspondingly, scattering and radiation damping of the disk increases, which are important aspects in the design of plasmonic nanostructures and their application in, for example, surface enhanced spectroscopies. Generally, combining EELS and CL with optical scattering and extinction spectroscopy provides a conceptual toolbox, relying on reciprocity to assess the full breadth of the properties of plasmonic modes.

\section{METHODS}

Silver nanodisks (30 $\mathrm{nm}$ thick) with diameters ranging from 150 to $500 \mathrm{~nm}$ were prepared by electron beam lithography in a RAITH eLINE+ system, using a poly(methyl methacrylate) resist on a $15 \mathrm{~nm}$ thick silicon nitride $\left(\mathrm{Si}_{3} \mathrm{~N}_{4}\right)$ membrane and a standard silver evaporation and lift off procedure. ${ }^{26}$ Silver was evaporated at a vacuum pressure of approximately $5 \times 10^{-6}$ mbar with an evaporation rate of $10 \AA / \mathrm{s}$ and no sample cooling.

The samples were studied in a VG HB 501 STEM, equipped with a cold field emission gun (energy full width at half maximum $<300 \mathrm{meV}$ ) at $100 \mathrm{keV}$ electron beam energy. Both, EELS and CL systems are homemade and fitted to the same microscope. ${ }^{16,27}$ The EELS system is based on a 666 Gatan spectrometer, fitted with custom made filter and multipole power supply and a direct, optical fiber free, optical coupling to a CCD camera. The CL system ${ }^{28}$ consists of a parabolic mirror that can be put into optical focus through a three axis high precision stage. Light reflected by the mirror is sent onto one end of an optical fiber bundle. The other end of the optical fiber is fitted to an optical spectrometer that is not fixed on the STEM column. All experiments on the same disks have been performed on the same day. As the acquisition parameters were very different, EELS and CL experiments have been made in sequences. All data presented in this work stem from spectral images. EELS spectra have been spectrally realigned and deconvoluted from the zero loss peak, using a Richardson-Lucy deconvolution algorithm (see Figure S5 in the Supporting Information). ${ }^{29}$ CL spectra have been converted from $\mathrm{nm}$ to $\mathrm{eV}$ units by applying a $\lambda^{2}$ prefactor (see Supporting Information, "Normalization" section).

The EELS and CL experiments were modeled by calculating numerically the electric field induced by a fast electron source moving perpendicular to single silver nanodisks lying on a $\mathrm{Si}_{3} \mathrm{~N}_{4}$ membrane. EELS spectra were obtained from the work done by the Lorentz force on the fast electron source (eq 8 from ref 30); CL spectra, from the radiant energy crossing the portion of a sphere surface located at infinity (eq 50 from ref $30)$. A fraction of the sphere surface $(1.2 \pi \mathrm{sr}$ of the upper half sphere, excluding the hole at the topmost position where the electron beam passes) was taken to mimic the actual detection angular spread used in the experiment (schematically shown in Figure 1a). The calculations were performed using the Boundary Element Method Maxwell equation solver ${ }^{31}$ available in the MNPBEM toolbox. ${ }^{32}$ The $\mathrm{Si}_{3} \mathrm{~N}_{4}$ membrane was modeled as a disk of relative permittivity $\varepsilon=4$ whose top facet is in contact with the bottom facet of the silver nanodisk. The relative permittivity of silver was taken from ref 33 . The nanodisk and substrate boundaries were meshed nonuniformly, with the smallest mesh elements around the nanodisk edges. Particles with different sizes were modeled by changing the nanodisk and substrate sizes while keeping the same mesh. Convergent simulations were obtained by taking the radius of the substrate disk $500 \mathrm{~nm}$ larger than the nanodisk radius and by using mesh elements with areas of $3 \mathrm{~nm}^{2}$. As an example, for a $200 \mathrm{~nm}$ large nanodisk, the nanodisk and substrate were discretized respectively by about 6000 and (excluding the boundary in contact with the nanodisk) 4500 elements. The edge spectra were calculated for an electron position within the disk located $5 \mathrm{~nm}$ away from the edge. The influence of the electron beam position, the damping, and the angular detection range on the simulated CL spectra is discussed in the Supporting Information (see "Agreement between simulation and experiment" section and Figure S4).

\section{AUTHOR INFORMATION}

\section{Corresponding Author}

*E mail: franz.schmidt@uni graz.at. ORCID

Franz-Philipp Schmidt: 0000000301252704

Mathieu Kociak: 0000000188580449

Notes

The authors declare no competing financial interest.

\section{ACKNOWLEDGMENTS}

This work was supported by the FWF SFB NextLite (F4905 N23), ESTEEM2 (FP7 project, no. 312483), NAWI Graz, and the Graz Centre for Electron Microscopy (ZFE). We thank Markus Krug and Gernot Schaffernak for fruitful discussions on optical spectroscopy of metallic nanoparticles.

\section{REFERENCES}

(1) García de Abajo, F. J.; Kociak, M. Probing the photonic local density of states with electron energy loss spectroscopy. Phys. Rev. Lett. 2008, 100, 106804.

(2) Hohenester, U.; Ditlbacher, H.; Krenn, J. R. Electron energy loss spectra of plasmonic nanoparticles. Phys. Rev. Lett. 2009, 103, 106801.

(3) Nelayah, J.; Kociak, M.; Stéphan, O.; García de Abajo, F. J.; Tencé, M.; Henrard, L.; Taverna, D.; Pastoriza Santos, I.; Liz Marzán, L. M.; Colliex, C. Mapping surface plasmons on a single metallic nanoparticle. Nat. Phys. 2007, 3, 348-353.

(4) Schaffer, B.; Hohenester, U.; Trügler, A.; Hofer, F. High resolution surface plasmon imaging of gold nanoparticles by energy filtered transmission electron microscopy. Phys. Rev. B: Condens. Matter Mater. Phys. 2009, 79, 56.

(5) Schmidt, F. P.; Ditlbacher, H.; Hohenester, U.; Hohenau, A.; Hofer, F.; Krenn, J. R. Dark plasmonic breathing modes in silver nanodisks. Nano Lett. 2012, 12, 5780-5783.

(6) Schmidt, F. P.; Ditlbacher, H.; Hohenester, U.; Hohenau, A.; Hofer, F.; Krenn, J. R. Universal dispersion of surface plasmons in flat nanostructures. Nat. Commun. 2014, 5, 3604.

(7) Campos, A.; Arbouet, A.; Martin, J.; Gérard, D.; Proust, J.; Plain, J.; Kociak, M. Plasmonic Breathing and Edge Modes in Aluminum Nanotriangles. ACS Photonics 2017, 4, 1257-1263. 
(8) Nicoletti, O.; La Peña, F. d.; Leary, R. K.; Holland, D. J.; Ducati, C.; Midgley, P. A. Three dimensional imaging of localized surface plasmon resonances of metal nanoparticles. Nature 2013, 502, 80-84.

(9) Collins, S. M.; Ringe, E.; Duchamp, M.; Saghi, Z.; Dunin Borkowski, R. E.; Midgley, P. A. Eigenmode Tomography of Surface Charge Oscillations of Plasmonic Nanoparticles by Electron Energy Loss Spectroscopy. ACS Photonics 2015, 2, 1628-1635.

(10) Hörl, A.; Haberfehlner, G.; Trügler, A.; Schmidt, F. P.; Hohenester, U.; Kothleitner, G. Tomographic imaging of the photonic environment of plasmonic nanoparticles. Nat. Commun. 2017, 8, 37.

(11) Kociak, M.; Zagonel, L. F. Cathodoluminescence in the scanning transmission electron microscope. Ultramicroscopy 2017, $176,112-131$.

(12) Yamamoto, N.; Araya, K.; García de Abajo, F. J. Photon emission from silver particles induced by a high energy electron beam. Phys. Rev. B: Condens. Matter Mater. Phys. 2001, 64, 1069.

(13) Losquin, A.; Kociak, M. Link between Cathodoluminescence and Electron Energy Loss Spectroscopy and the Radiative and Full Electromagnetic Local Density of States. ACS Photonics 2015, 2, 1619-1627.

(14) Kociak, M.; Stéphan, O. Mapping plasmons at the nanometer scale in an electron microscope. Chem. Soc. Rev. 2014, 43, 38653883.

(15) Coenen, T.; Schoen, D. T.; Mann, S. A.; Rodriguez, S. R. K.; Brenny, B. J. M.; Polman, A.; Brongersma, M. L. Nanoscale Spatial Coherent Control over the Modal Excitation of a Coupled Plasmonic Resonator System. Nano Lett. 2015, 15, 7666-7670.

(16) Losquin, A.; Zagonel, L. F.; Myroshnychenko, V.; Rodriguez Gonzalez, B.; Tence, M.; Scarabelli, L.; Forstner, J.; Liz Marzan, L. M.; Garcia de Abajo, F. J.; Stephan, O.; Kociak, M. Unveiling nanometer scale extinction and scattering phenomena through combined electron energy loss spectroscopy and cathodolumines cence measurements. Nano Lett. 2015, 15, 1229-1237.

(17) Kawasaki, N.; Meuret, S.; Weil, R.; Lourenço Martins, H.; Stéphan, O.; Kociak, M. Extinction and Scattering Properties of High Order Surface Plasmon Modes in Silver Nanoparticles Probed by Combined Spatially Resolved Electron Energy Loss Spectroscopy and Cathodoluminescence. ACS Photonics 2016, 3, 1654-1661.

(18) Bosman, M.; Ye, E.; Tan, S. F.; Nijhuis, C. A.; Yang, J. K. W.; Marty, R.; Mlayah, A.; Arbouet, A.; Girard, C.; Han, M. Y. Surface plasmon damping quantified with an electron nanoprobe. Sci. Rep. 2013, 3, 1312.

(19) Martin, J.; Kociak, M.; Mahfoud, Z.; Proust, J.; Gerard, D.; Plain, J. High resolution imaging and spectroscopy of multipolar plasmonic resonances in aluminum nanoantennas. Nano Lett. 2014, $14,5517-5523$.

(20) Fung, K. H.; Kumar, A.; Fang, N. X. Electron photon scattering mediated by localized plasmons: A quantitative analysis by eigen response theory. Phys. Rev. B: Condens. Matter Mater. Phys. 2014, 89, 45408.

(21) Miao, X.; Guo, K.; Qian, C.; Wang, J.; Zhao, D.; Fung, K. H. Electron beam excited photon emission from monopole modes of a plasmonic nano disc. Opt. Lett. 2017, 42, 3387.

(22) Langhammer, C.; Kasemo, B.; Zoric, I. Absorption and scattering of light by $\mathrm{Pt}, \mathrm{Pd}, \mathrm{Ag}$, and $\mathrm{Au}$ nanodisks: absolute cross sections and branching ratios. J. Chem. Phys. 2007, 126, 194702.
(23) Hohenester, U.; Trügler, A. MNPBEM A Matlab toolbox for the simulation of plasmonic nanoparticles. Comput. Phys. Commun. 2012, 183, 370-381.

(24) Krug, M. K.; Reisecker, M.; Hohenau, A.; Ditlbacher, H.; Trügler, A.; Hohenester, U.; Krenn, J. R. Probing plasmonic breathing modes optically. Appl. Phys. Lett. 2014, 105, 171103.

(25) Coenen, T.; Bernal Arango, F.; Femius Koenderink, A.; Polman, A. Directional emission from a single plasmonic scatterer. Nat. Commun. 2014, 5, 3250.

(26) Hohenau, A.; Ditlbacher, H.; Lamprecht, B.; Krenn, J. R.; Leitner, A.; Aussenegg, F. R. Electron beam lithography, a helpful tool for nanooptics. Microelectron. Eng. 2006, 83, 1464-1467.

(27) Myroshnychenko, V.; Nelayah, J.; Adamo, G.; Geuquet, N.; Rodriguez Fernandez, J.; Pastoriza Santos, I.; MacDonald, K. F.; Henrard, L.; Liz Marzan, L. M.; Zheludev, N. I.; Kociak, M.; Garcia de Abajo, F. J. Plasmon spectroscopy and imaging of individual gold nanodecahedra: a combined optical microscopy, cathodolumines cence, and electron energy loss spectroscopy study. Nano Lett. 2012, 12, 4172-4180.

(28) Kociak, M.; Zagonel, L. F.; Tence, M.; Mazzucco, S. Adjustable cathodoluminescence detection system and microscope employing such a system. 2014.

(29) Gloter, A.; Douiri, A.; Tence, M.; Colliex, C. Improving energy resolution of EELS spectra: an alternative to the monochromator solution. Ultramicroscopy 2003, 96, 385-400.

(30) García de Abajo, F. J. Optical excitations in electron microscopy. Rev. Mod. Phys. 2010, 82, 209-275.

(31) García de Abajo, F. J.; Howie, A. Retarded field calculation of electron energy loss in inhomogeneous dielectrics. Phys. Rev. B: Condens. Matter Mater. Phys. 2002, 65, 4156.

(32) Hohenester, U. Simulating electron energy loss spectroscopy with the MNPBEM toolbox. Comput. Phys. Commun. 2014, 185, 1177-1187.

(33) Palik, E. Handbook of Optical Constants of Solids; Academic Press, 1985. 